\title{
COVID-19 and Older Adults. Lessons Learned from the Italian Epicenter
}

\author{
Matteo Cesari, MD, PhD ${ }^{1,2}$ and Manuel Montero-Odasso, MD, PhD, AGSF, FGSA, FRCPC $3,4,5$ \\ ${ }^{1}$ Department of Clinical Sciences and Community Health, Università degli Studi di Milano, Milan; \\ ${ }^{2}$ Geriatrics Unit, Fondazione IRCCS Ca'Granda Ospedale Maggiore Policlinico, Milan, Italy; ${ }^{3}$ Schulich School \\ of Medicine \& Dentistry, Department of Medicine and Division of Geriatric Medicine, The University of Western \\ Ontario, London, ON; ${ }^{4}$ Department of Epidemiology and Biostatistics, The University of Western Ontario, \\ London, ON; ${ }^{5}$ Gait and Brain Lab, Parkwood Institute, Lawson Health Research Institute, London, ON, Canada
}

https://doi.org/10.5770/cgj.23.445

\begin{abstract}
On March 13th, 2020, The World Health Organization effectively established that Europe is the new the COVID-19 pandemic world epicenter, as cases in Italy and other European nations soared. The numbers in Italy have climbed with over 80,000 cases as of March 25th, 2020 and with over 8000 deaths, placing Italy now as the country with the highest mortality rate. Importantly, older adults are particularly vulnerable to get severe illness, complications, and to have a higher mortality rate than any other age group. The clinical presentation in older adults with severe illness, in the experience from geriatricians in Lombardy, is described as quite sudden; patients can develop severe hypoxemia with the need of ventilation support in few hours. Geriatric syndromes are not a common form of presentation for COVID-19 in severe illness. It is suggested that stratification by frailty level may help to detect the most vulnerable, and decisions about healthcare resource prioritization should not be taken based only on age itself or previous diagnosis, such as having dementia.
\end{abstract}

Key words: COVID-19, pandemic, aged, older adults, Italy

"They asked for my availability and I said yes. When you decide to be a doctor in life, you get involved. I swore an oath. Afraid of getting sick? Then it is better not to be a doctor." March 2020

- Dr Giampiero Giron, 85 years old - Professor emeritus, University of Padua

\section{INTRODUCTION}

This quote from Dr Giampiero Giron, an 85-year-old Italian anesthetist and member of the team that performed Italy's first heart transplant, encapsulates the commitment of doctors in difficult times and also the importance of older adults in our societies. On March 13th, 2020, the World Health Organization (WHO) effectively established that Europe is the new the COVID-19 pandemic epicenter of the world, as cases in Italy and other nations on the continent soared. As of March 26th 2020 , across the world, cases reached over 500,000 and deaths reached 23,000. ${ }^{(1)}$ This dramatic and exponential increase in the prevalence and deaths related to COVID-19 is spreading rapidly through Europe, as it is now reporting more cases and deaths than the rest of the world combined.

Specifically, the numbers in Italy have climbed, with over 80,000 cases and 8,000 deaths as of March 26th, 2020, placing Italy now as the country with the highest mortality rate ${ }^{(1)}$ Importantly, older adults are particularly vulnerable to get severe illness and complications from this disease, and they also have a higher mortality rate than any other age group. The case fatality rate in Italy jumped from the $0.8 \%$ in those 50 to 59 years of age, to $2.7 \%$ in those 60 to 69 years of age, and to $21.1 \%$ in those 90 years and older (Table 1). Dr Matteo Cesari, Professor and Chair of Geriatric Medicine of the University of Milan, replied to our questions on March 26th, 2020, which are transcribed below.

\section{Current Situation in Italy}

MMO: Thank you, Matteo, for making this time for our questions. We know you are under extreme pressure and busy with unprecedented clinical demands, therefore we appreciate you providing us with direct information. How is the situation in Lombardy and, specifically, Milan, now (March 25, 2020)?

MC: The situation in Milan is still very complicated. We have not yet reached the peak of the number of new COVID cases, which is expected to arrive in the very next few days.

Figure 1 shows the distribution of cases among Italian regions, and it can be seen how the north of Italy took the first hit. The hospitals have been struggling since the beginning of the crisis because they needed to substantially redraw their 
TABLE 1.

Istituto Svperiore di Sanitá (ISS) data on cases that tested positive for SARS-COV-2 infection diagnosed

by all Italian Regions/Autonomous Provinces

\begin{tabular}{ccc}
\hline $\begin{array}{c}\text { Age } \\
\text { (years) }\end{array}$ & $\begin{array}{c}\text { Deaths } \\
(n(\%)]\end{array}$ & $\begin{array}{c}\text { Case Fatality } \\
\text { Rate (CFR) }\end{array}$ \\
\hline $0-9$ & $0(0 \%)$ & $0 \%$ \\
$10-19$ & $0(0 \%)$ & $0 \%$ \\
$20-29$ & $0(0 \%)$ & $0 \%$ \\
$30-39$ & $2(0.2 \%)$ & $0.2 \%$ \\
$40-49$ & $4(0.4 \%)$ & $0.2 \%$ \\
$50-59$ & $24(2.4 \%)$ & $0.8 \%$ \\
$60-69$ & $77(7.6 \%)$ & $2.7 \%$ \\
$70-79$ & $360(35.4 \%)$ & $10.8 \%$ \\
$80-89$ & $438(43.0 \%)$ & $17.5 \%$ \\
$>90$ & $96(9.4 \%)$ & $21.1 \%$ \\
Not reported & $16(1.6 \%)$ & $2.8 \%$ \\
Total & $1,017(100 \%)$ & $6.2 \%$ \\
\hline
\end{tabular}

pathways in an attempt to isolate COVID-positive patients from the others. At the same time, the system was not ready to sustain the massive number of patients with respiratory symptoms arriving all together at the emergency departments.
As we can see in Figure 2, 25\% of our patients presented with severe disease and 5\% are critical, all of them require hospitalization. This, combined with the shortage of ICU beds and the gradual decrease in health-care professionals that were falling sick, has made things extremely difficult.

MMO: How is the clinical presentation of the symptomatic disease in older adults? Have you found any differences when compared with younger adults? Do older patients with severe COVID-19 illness present with severe dyspnea and hypoxemia, as do the younger adults? Or do older patients present with geriatric syndromes, such as delirium?

MC: The clinical manifestation is usually characterized by fever and cough. It might appear as a normal pneumonia. In fact, very frequently, the COVID-19 case manifests itself as a pneumonia resistant to antibiotics and standard care. The worsening of respiratory symptoms is today considered a clear sign suggestive of COVID infection. Since the very beginning, we have tried as much as possible to isolate patients with respiratory symptoms or flu-like manifestations from the others, given the high contagiousness of the coronavirus. This was also to allow the time to test for the possible SARS-CoV-2 infection. In this context, however, things are made difficult by the relatively high number of false-negative cases found from the nasopharyngeal swab. For this reason, people tend to be kept isolated even when tested negative, if the clinical suspicion remains high.

Overall, the Istituto Superiore di Sanitá (ISS) from Italy reports that the most common complications are the following: acute respiratory distress syndrome $(97.5 \%$ of cases $)$,

\section{Total number of COVID-19 cases diagnosed by the Italian Regional Reference Laboratories}

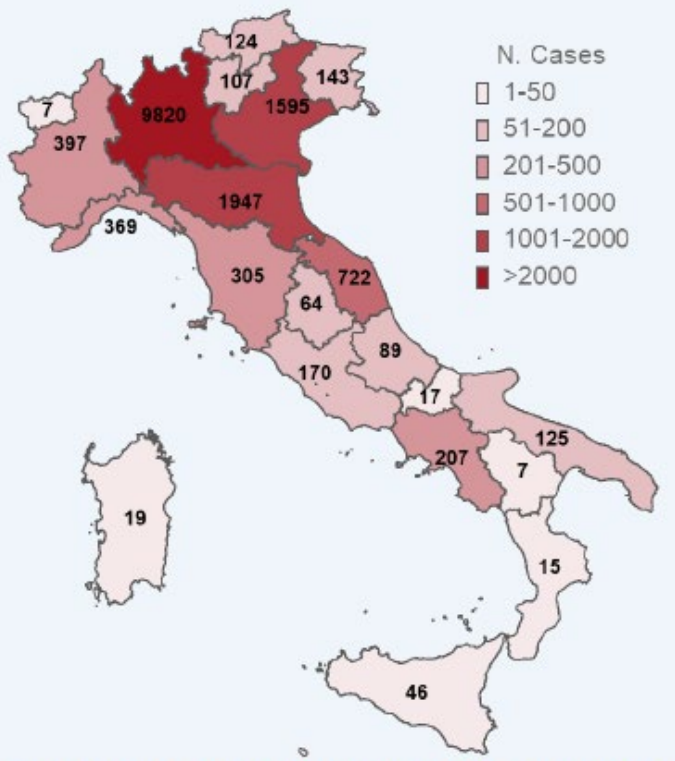

By Region/Autonomous Provinces of diagnosis

(data available only for 16,295 )

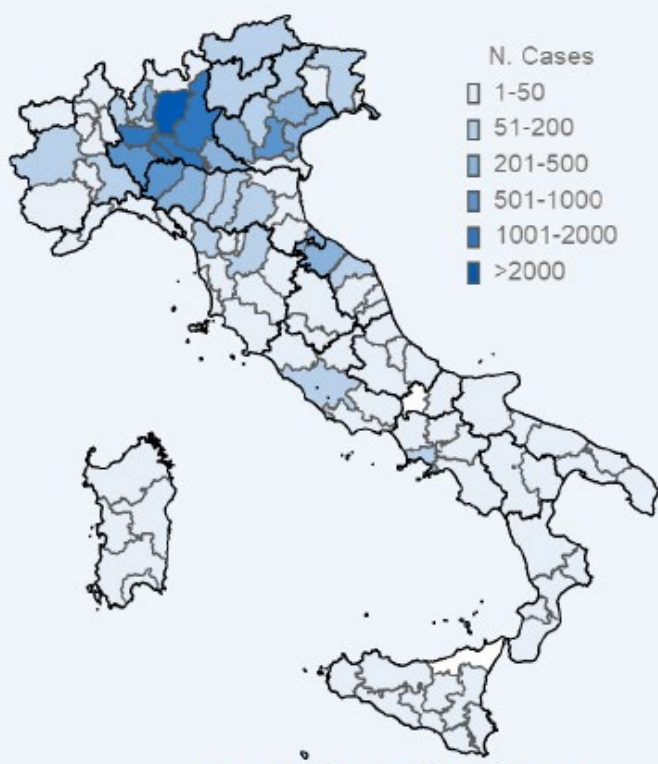

By province of residence

(data available only for 15,352)

FIGURE 1. Number of COVID-19 cases regionally in Italy 


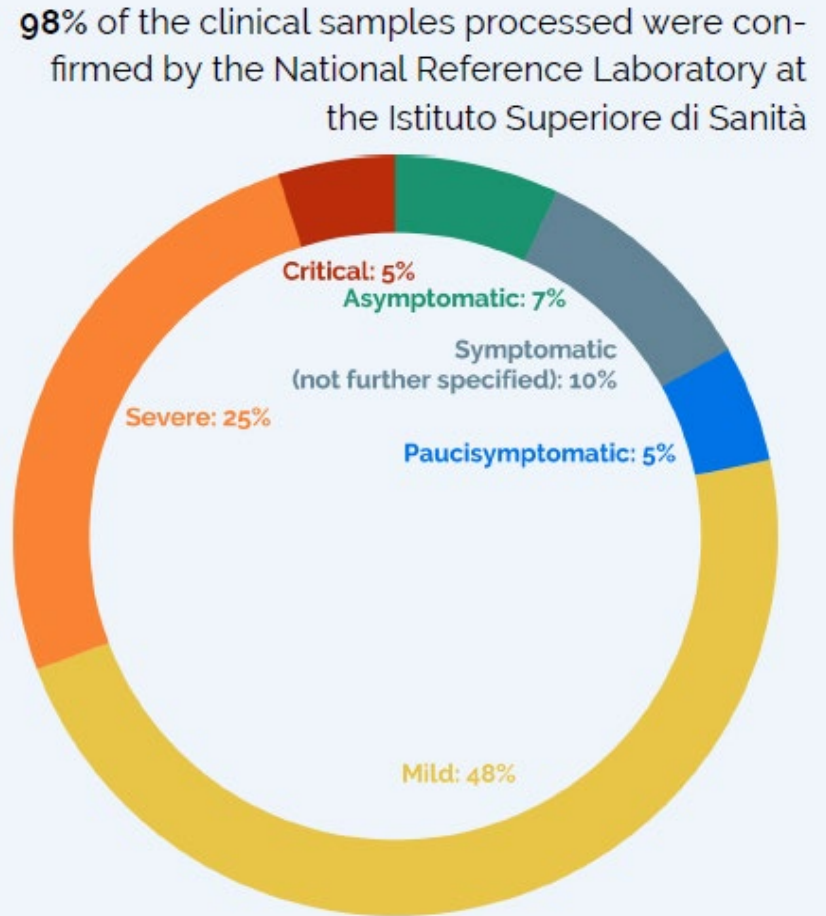

Data available only for 6,941 cases

FIGURE 2. Percentage of clinical presentation of patients who tested COVID-19-positive

acute renal failure $(28.9 \%)$, acute cardiac injury (11.2\%), and superinfection (10.2\%). Nevertheless, the clinical manifestation of the COVID in older persons can be associated with delirium. The main difference is the unexpectedly rapid course of the disease. Many patients convert in 20-30 min from relatively stable conditions into severe respiratory distress. In other words, preliminary signs can be there, but their identification and management is often impossible given the quick evolution of the case.

It is also noteworthy that hospitals have relatively few geriatricians, and they had to reorganize their workforces creating mixed teams. This was needed to face the shortage of personnel. I have to say that, in this emergency situation, it is very difficult for non-specialized clinicians to pay attention and detect geriatric syndromes that are largely overlooked and underestimated even in normal times.

\section{Current Situation in Hospital Settings}

MMO: How are access to Emergency Room (ER) care, inpatient beds, and critical care beds being managed? There have been reports in the media that older citizens should get a lower priority for access to medical services that are now limited, such as intensive care facilities and ventilation. This begs the question of whether this practice should go unchallenged as a form of "pragmatism", or whether we should think through how to engage with this in order to at least reduce the risk of the most egregious forms of discrimination.
MC: ERs have been exponentially overwhelmed over the past weeks. The shortage of beds, especially in ICUs, has sometimes led to very difficult decisions. The relatively few number of ventilators, in fact, has generated discussions about the ethics of procedures applied to certain patients. The Italian Society of Analgesia, Anaesthesia, Resuscitation, and Intensive Care (SIAARTI) released a document recommending a triage in a low-resource scenario based on age, comorbidities, and functional status.(2) Unfortunately, the ageistic approach diffused in our society and in traditional medicine, as well as the poor knowledge about how to operationally apply these recommendations, makes critical decisions still (too largely) governed by the age criterion. I do not/cannot justify these decisions, but we also need to consider that the rapid course of the disease leads to taking quite rapid decisions.

MMO: Related to the previous question, in the stratification or triage of sick patients to maximize health resources, do you use a functional scale, or frailty status scale, or dementia severity scale for clinical decisions?

MC: Age of the patient itself has been the easiest parameter to obtain in an emergency to decide on allocation of care. It is wrong and we know it. Unfortunately, our system does not yet consider more robust and reliable alternatives for measuring the resilience of the person. Perhaps, we should have thought about nesting more geriatric principles (i.e., meaning of the disease construct at old age, importance of functions) in other specialties. The simple adoption of the Clinical Frailty Scale measuring the pre-illness status of the patient might have already represented an important parameter in order to take better decisions. ${ }^{(3-5)}$

MMO: How does a COVID unit look like in Italy for geriatricians?

MC: A COVID unit is very far from our usual geriatric world. The communication between health-care professionals and patients is extremely limited by the personal protective equipment (PPE), as well as by the patient's clinical conditions and respiratory devices. The unit environment is not age-friendly; its design is not oriented to individuals with sensory deficits or cognitive and/or mobility impairment. These units are busy, noisy, and only distinguish the "dirty" areas from the "clean" ones. Family members are not allowed to enter the unit. Therefore, patients have no contact with them, and family members are usually not receiving news of their relatives. For this reason, we have volunteers who are regularly keeping families updated about the status of the patients over the phone. We try to maintain such contacts, knowing that the worst thing happening with COVID is the isolation of the person.

\section{The Situation in Long-term Care Facilities}

MMO: How is the situation in long-term care facilities? Do you have any recommendations to provide - particularly in this setting? 
MC: Long term facilities have initially tried to contain the diffusion of the virus within their facilities by reducing/ closing admissions from the hospitals. Suspected cases have been isolated and/or sent to the emergency units in order to guarantee the safety of the rest of the residents. The work in long-term care is not easy these days, also because resources to test patients (and personnel) remain limited. There have also been limitations in some cases of protected personal equipment's (PPEs). Just in these last few days, the WHO has released recommendations for the management of COVID in long term care. (https://apps.who.int/iris/bitstream/handle/ 10665/331508/WHO-2019-nCoV-IPC_long_term_care2020.1-eng.pdf) ${ }^{(6)}$

\section{Medication Access for Older Adults}

MMO: As many older adults suffer from chronic conditions requiring medication, the question of how medication access is being managed is of paramount importance. How are you managing medication access to older patients with chronic conditions?

MC: Outpatient clinics were closed at the very beginning of the coronavirus outbreak. Only visits that could not be postponed or that met urgency criteria were maintained. A lot of older persons have found themselves completely isolated from medications, but also from the simple access to groceries. In this scenario, consider that general practitioners have found themselves in huge difficulties (shortage of PPEs, a lot of cases of COVID, some even died). The consequent shortage of primary care physicians has further complicated things.

Frail older persons with chronic diseases will likely pay a huge toll for this coronavirus pandemic, even if not directly infected by it. This emergency situation has destroyed our already weak integration of care services, critical for the health of frail individuals.

\section{Potential Explanations for the Higher Prevalence and Severity of the COVID-19 Crisis in Older Adults}

MMO: An additional fact is that the case mortality rate is higher in older adults, but particularly in those with cardiovascular disease, hypertension, and diabetes or having more than three comorbidities (Table 2). It has been suggested that Angiotensin-converting enzyme 2 (ACE-2) inhibitors may explain the higher risk and severity of the disease in older $\operatorname{adults}^{(7)}$ because they increase the up-regulation of ACE-2 receptor expression - the receptor that the SARS CoV-2 virus uses to enter the human cells. ${ }^{(7)}$ Are clinicians changing the ACE-2 inhibitor medication in their patients for another medication such as calcium channel blockers?

MC: There have been many news items around about the positive or negative effects of certain medications in face of the COVID. To my knowledge, most of the clinicians have not changed the prescriptions of cardiovascular drugs of their patients, also given the recommendations from the American Heart Association, the Heart Failure Society of America, and
TABLE 2.

Most common comorbidities observed in COVID-19-positive deceased patients by type of disease ${ }^{a}$

\begin{tabular}{lcc}
\hline \multicolumn{1}{c}{ Disease } & $N$ & $\%$ \\
\hline Ischemic heart disease & 126 & 24.5 \\
Atrial fibrillation & 121 & 23.5 \\
Stroke & 64 & 12.5 \\
Hypertension & 384 & 74.7 \\
Diabetes & 157 & 30.5 \\
Dementia & 90 & 17.5 \\
COPD & 98 & 19.1 \\
Active cancer in the past 5 years & 92 & 17.9 \\
Chronic liver disease & 25 & 4.9 \\
Chronic renal failure & 119 & 23.2 \\
\hline
\end{tabular}

\begin{tabular}{lcc}
\hline \multicolumn{1}{c}{ Number of Comorbidities } & & \\
\hline 0 comorbidities & 7 & 1.4 \\
1 comorbidities & 110 & 21.4 \\
2 comorbidities & 134 & 26.1 \\
3 comorbidities and over & 263 & 51.2 \\
\hline
\end{tabular}

a Data on diseases were based on chart review and was available on 514 patients dying in-hospital for whom it was possible to analyze clinic charts (15.0\% of the sample). Mean number of diseases was 2.7 (median 3, SD 1.6). Overall, $1.4 \%$ of the sample presented with no comorbidities, $21.4 \%$ with a single comorbidity, $26.1 \%$ with 2 , and $51.2 \%$ with 3 or more.

the American College of Cardiology. Another news item was related to a possible association of ibuprofen to negative outcomes in COVID cases. This led to the suggestion of using paracetamol over ibuprofen. In this other case, the WHO explained that the news was not supported by evidence and discouraged the non-utilization of ibuprofen.

MMO: Is there something else that you want to add?

MC: Thank you, Manuel, for reaching out and I hope our experience will help Canadian geriatricians and health-care workers dealing with older adults during this overwhelming pandemic. We are in uncharted territory.

\section{KEY POINTS}

1. Older adults present the highest mortality rate in this pandemic, in the Italian epicenter and worldwide.

2. Clinical presentation in older adults with severe illness is quite sudden; patients can develop severe hypoxemia and need ventilation support in very few hours.

3. Geriatric syndromes are overlooked as a form of presentation of COVID-19 severe illness, being this dominated by severe respiratory symptoms. 
4. Stratification by frailty level may help to early detect the most vulnerable.

5. Decision concerning health-care utilization resources should not be taken based only on age itself or isolated diagnoses, such as the presence of dementia; rather, advanced directives or functional/frailty stratification may be considered.

\section{CONFLICT OF INTEREST DISCLOSURES}

The authors declare that no conflicts of interest exist.

\section{REFERENCES}

1. John Hopkins University. Coronavirus Resource Center. Baltimore, MD: Bloomberg School of Public Health; 2020. Available from: https://coronavirus.jhu.edu

2. SIAARTI. Clinical ethics recommendations for the allocation of intensive care treatments, in exceptional, resource-limited circumstances [Internet]. Rome, Italy: SIAARTI; 2020.
3. Rockwood K, Song X, MacKnight C, et al. A global clinical measure of fitness and frailty in elderly people. CMAJ. 2005;173(5):489-95.

4. Cesari M, Gambassi G, van Kan GA, et al. The frailty phenotype and the frailty index: different instruments for different purposes. Age Ageing. 2014;43(1):10-12.

5. Juma S, Taabazuing MM, Montero-Odasso M. Clinical Frailty Scale in an acute medicine unit: a simple tool that predicts length of stay. Can Geriatr J. 2016;19(2):34-39.

6. The World Health Organization. Infection prevention and control guidance for long-term care facilities in the context of COVID-19: interim guidance, 21 March. Geneva, Switzerland: Institutional Repository for Information Sharing (iris); 2020.

7. Fang L, Karakiulakis G, Roth M. Are patients with hypertension and diabetes mellitus at increased risk for COVID-19 infection? Lancet Respir Med. 2020;8(4):e21.

Correspondence to: Manuel Montero-Odasso, MD, PhD, AGSF, FGSA, FRCPC, Schulich School of Medicine and Dentistry, The University of Western Ontario, 550 Wellington Rd., Room A3-116, London, ON N6C 0A7, Canada

E-mail: mmontero@uwo.ca 